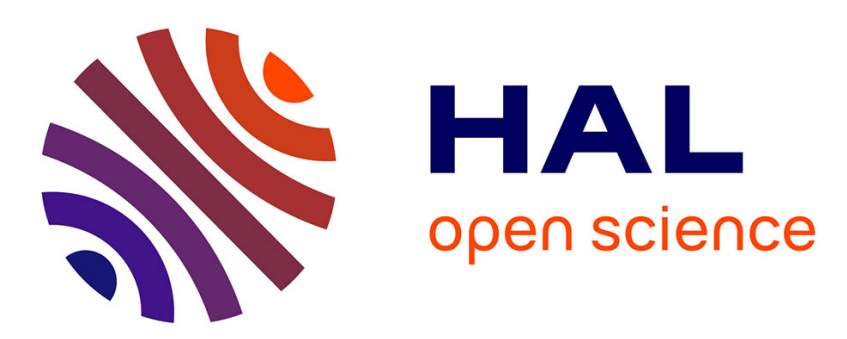

\title{
Enseigner la lecture intime du texte littéraire grâce à l'édition hypertextuelle
}

\author{
Thierry Soubrié
}

\section{To cite this version:}

Thierry Soubrié. Enseigner la lecture intime du texte littéraire grâce à l'édition hypertextuelle. Document numérique - Revue des sciences et technologies de l'information. Série Document numérique, 2001, 5 (1-2), pp.181-208. 10.3166/dn.5.1-2.181-208 . hal-02509513

\section{HAL Id: hal-02509513 https://hal.science/hal-02509513}

Submitted on 17 Mar 2020

HAL is a multi-disciplinary open access archive for the deposit and dissemination of scientific research documents, whether they are published or not. The documents may come from teaching and research institutions in France or abroad, or from public or private research centers.
L'archive ouverte pluridisciplinaire HAL, est destinée au dépôt et à la diffusion de documents scientifiques de niveau recherche, publiés ou non, émanant des établissements d'enseignement et de recherche français ou étrangers, des laboratoires publics ou privés. 


\title{
Enseigner la lecture intime du texte littéraire grâce à l'édition hypertextuelle
}

\author{
Thierry Soubrié \\ Laboratoire DIDAXIS (DIPRALANG) \\ Université Paul Valéry \\ Route de Mende \\ 34199 Montpellier cedex 5 \\ Thierry.Soubrie@univ-montp3.fr
}

RÉSUMÉ. Ce travail se situe à la croisée de trois grands domaines : l'informatique éducative, la didactique de la littérature et la lecture. L'informatique, et en particulier la technologie de l'hypertexte, permettent désormais, au travers de dispositifs de lecture électronique, d'appréhender les textes dans leur épaisseur intra, extra et intertextuelle. Après les théories de la réception, ces nouveaux environnements réaffirment à leur manière l'interactivité de la lecture. Lire n'est pas une activité linéaire mais donne lieu à de multiples actions sur le texte: comparaisons, rapprochements, annotations, etc. Transposée dans le champ de l'enseignement/apprentissage de la lecture littéraire, cette réflexion est le fondement d'une expérience visant à démontrer que l'édition hypertextuelle d'un texte en classe peut aider, mieux que tout autre moyen, à faire prendre conscience aux élèves de la nature intime de la lecture littéraire.

ABSTRACT.This work lies at the border of three main fields: educational information technology, literature didactics and text reading. Information technology and specifically the technology of hypertext now make it possible, through electronic reading devices, to embrace texts in their intra, extra and intertextual dimensions. After the theories of reception, these new technological environments bring out in their own way the interactive character of reading. Reading is not a linear process but submits the text to numerous actions : comparisons, connections, annotations, etc. Once transposed in the area of teaching/learning of literary reading, this reflexion is the basis of an experiment which aims at proving that publishing a text at school in the form of an hypertext can help students, better than any other means, to become aware of the intimate nature of literary reading.

MOTS-CLÉs: informatique, lecture, hypertexte, didactique de la littérature, sémiologie, psychologie cognitive, théories littéraires, école primaire, enseignement/apprentissage de la lecture, écrilecture.

KEYWORDS: : information technology, reading, hypertext, cognitive psychology, literature theories, primary school, teaching/learning of reading.

Document numérique. Volume $5-\mathrm{n}^{\circ} 1-2 / 2001$, pages 181 à 208 


\section{Apparition d'un nouveau comportement de lecteur}

A partir du moment où le niveau technologique a permis la numérisation des documents, le rapport à l'écrit a irrémédiablement changé. Manipulable, le texte a vite perdu son caractère d'intangibilité, voire dans le cas du texte littéraire sa quasisacralité. Codés sous la forme d'une suite de chiffres, les mots deviennent l'objet de toutes sortes de traitements informatiques : établissement de listes de concordances, repérage des mots, collationnement de textes, etc. Les index hiérarchiques (listes des formes contenues dans les romans classées par ordre de fréquence) donnent souvent des indications thématiques précieuses - dans Les Misérables, les premiers substantifs de la liste sont « homme», « Marius » et «Jean » [BER 99] -, les listes de formes hors ou en contexte apportent des informations d'ordre stylistique, métrique ou autre. Des lois statistiques comme celle de l'écart réduit ou celle du calcul des spécificités rendent possible la comparaison du vocabulaire de corpus de taille différente. Même s'il convient, dès lors que l'on soumet un texte littéraire à des comptages, de manier les résultats avec précaution.

Il est vrai que certaines de ces pratiques existaient avant l'arrivée de l'informatique. Michel Bernard rappelle en effet que des concordances et des index sont fabriqués depuis le Moyen Age et que l'on a «pratiqué une forme manuelle de la statistique textuelle depuis le XIXe siècle» [BER 99]. Mais l'informatique apporte la possibilité de traiter des corpus longs et de décharger le chercheur des tâches les plus fastidieuses. Il permet surtout, progressivement, d'étendre ces pratiques à un public de plus en plus large. Grâce, non seulement à Internet (bibliothèques virtuelles), mais aussi à l'arrivée sur le marché de dispositifs de lecture électronique (postes de lecture de la Bibliothèque nationale de France et ebooks).

Une des conséquences de cette technicisation est l'apparition d'un nouveau comportement de lecteur. Par ses caractéristiques physiques mêmes, un écran pour lire et un clavier pour écrire, l'ordinateur enjoint en quelque sorte le lecteur à exercer une action véritablement concrète sur le texte. Il s'agit non plus seulement de lire, mais aussi d'écrire. Effectuer des recherches lexicales et statistiques, comme on vient de la voir, mais également insérer et gérer des annotations, parcourir, voire créer, des réseaux de liens inter, extra et intratextuels. Bien que ces activités n'impliquent pas forcément un travail de production écrite, elles mettent à tout le moins en œuvre une réflexion métatextuelle et métalinguistique, où lire devient assumer la position du scripteur.

Dans un récent ouvrage intitulé Littérature, Informatique, Lecture [VUI 99], deux auteurs ${ }^{1}$, tous deux chercheurs au Centre d'études et de recherches sur les

\footnotetext{
${ }^{1}$ Il s'agit d'Alain Vuillemin [VUI 00] et d'Arnaud Gillot [GIL 00].
} 
textes électroniques (CERTEL), désignent ce nouveau comportement de lecteur, où la lecture est consubstantielle à l'écriture, par le terme d' «écrilecture », néologisme forgé en 1992 par Pedro Barbosa dans une thèse sur la création littéraire et l'ordinateur.

Dans cette évolution, l'hypertexte joue un rôle important. Grâce à sa structure réticulaire et cognitive [SOU 01], l'accès aux savoirs ne se fait plus de façon unilatérale, linéaire, mais multiple, au travers d'éclairages différents.

La génétique des textes a très bien compris ce qu'elle pouvait tirer de cette nouvelle rhétorique. Jean-Louis Lebrave rapporte ainsi, qu'à l'opposé du modèle philologique classique qui occultait, au nom d'une «idéologie romantique de l'inspiration », le versant de la production des œuvres, l'hypertexte permet de restituer « aux voix et aux mains la plénitude d'une créativité à part entière » [LEB 97]. Affranchie de l'ordre linéaire de l'imprimé, l'œuvre se retrouve placée dans l'hypertexte à la croisée de l'avant-texte. Et inversement.

Alain Vuillemin distingue trois types d'écrilecture. Elle est périphérique lorsqu'il s'agit d'apporter des annotations, elle est centrale, fondatrice, dès lors que le texte devient préhensible, c'est-à-dire lorsque, numérisé, il devient manipulable, interrogeable; elle est enfin créatrice quand l'ordinateur est au service de l'extraction des aspects méconnus d'un texte, lorsqu'il devient possible de «décomposer les éléments qui le [texte] constituent et d'en découvrir la nature intime pour mieux en apprécier les significations et la portée » [VUI 99 : 106]. En fait, il s'agit plus généralement dans ce dernier cas de figure, non pas d'appréhender un texte mais un ensemble de textes fortement solidaires (un architexte), à l'aide de multiples modes de lecture interactive (procédures de recherche) et assistée (annotations).

Pour les tenants des théories de la réception, ce type de lecture, que certains qualifient de savante, n'est ni nouveau ni exceptionnel. L'interaction entre le texte et le lecteur est constitutive de l'acte de lecture. Un texte ne s'imprime pas automatiquement dans la conscience du lecteur. «La structure du texte et celle de l'acte de lecture sont complémentaires pour donner lieu à la communication » [ISE $76: 197]$.

Dans la phénoménologie de la lecture d'Iser, la position du lecteur dans le texte se situe au point d'intersection entre «protention », émission d'hypothèses, attentes, et rétention. Umberto Eco parle de «prévisions » et de «promenades inférentielles ». Lire en effet, ce n'est pas seulement dérouler du linéaire, c'est aussi s'arrêter, revenir en arrière, comparer, voire annoter.

Le travail de Barthes sur la nouvelle Sarrazine de Balzac [BAR 70] ou sur La vérité sur le cas de M. Valdemar de Poe [BAR 73], est la trace de ce travail de lecture : fragmentation du texte, comparaison des unités de lecture ainsi mises à jour (Iser parle de «corrélations de phrases »), rapprochement de certaines d'entre elles, projetant ainsi sur le texte différents points de vue interprétatifs, ajout de commentaires. 
Les dispositifs de lecture électronique constituent donc ni plus ni moins ce que l'on pourrait appeler, à l'instar de Pierre Lévy, des appareillages de lecture artificielle :

«Si lire consiste à sélectionner, à schématiser, à construire un réseau de renvois internes au texte, à associer à d'autres données, à intégrer les mots et les images à une mémoire personnelle en reconstruction permanente, alors les dispositifs hypertextuels constituent bel et bien une sorte d'objectivation, d'extériorisation, de virtualisation des processus de lecture $»[\mathrm{LEV}]$.

Précisons seulement que si la technologie informatique prend appui sur des processus cognitifs et intellectuels avérés, il n'en demeure pas moins qu'elle en décuple les possibilités. Dans l'histoire des modes de pensée, l'apparition de l'écrit, puis son émancipation progressive de l'ordre linéaire de l'oral (passage du volumen au codex, apparition des outils de lecture) a permis à un certain nombre d'opérations intellectuelles de se développer «comme la comparaison des énoncés, la hiérarchisation des éléments du discours, la réorganisation de la pensée, la migration des concepts, etc. » [CLE 98]. Les dispositifs de lecture électronique, en tant que «technologie intellectuelle» [GOO 79] nouvelle, s'inscrivent dans ce mouvement : traitement de corpus de taille importante, rapidité d'exécution des tâches, rôle des représentations graphiques, etc. Par ailleurs, l'architecture hypertextuelle de ces dispositifs permet d'appréhender les documents dans leur intégrité, et ce avec plus d'intelligibilité que ne le ferait un agencement hiérarchique des informations. Un hypertexte est en effet un réseau à l'intérieur duquel les relations entre les nœuds apportent autant d'informations que les nœuds eux-mêmes de façon à multiplier la valeur informative apportée par la simple somme des données prise comme référence [PAM 97].

\section{L'écrilecture à l'école}

C'est dans ce contexte qu'il m'est très rapidement apparu que ces nouveaux environnements de lecture, parce qu'ils matérialisent des opérations et des processus abstraits, pouvaient se révéler plus performants, pour sensibiliser les élèves à la nature fondamentalement interactive de la lecture littéraire, que des méthodes traditionnelles qui ne laissent bien souvent qu'entrevoir le phénomène. Comme le remarquaient il y a déjà six ans Bernard Stiegler et Gwendal Auffret à propos d'un dispositif de leur invention, LECAO (Lecture et Ecriture Critique Assistée par Ordinateur):

«Auparavant, la lecture d'un texte s'expliquait seulement, maintenant, il est possible de la montrer $\gg$ [AUF 96].

Des expériences ont déjà été menées dans ce sens, mais essentiellement tournées vers l'écriture. Scripertexte par exemple, logiciel d'aide à l'écriture élaboré par 
Jacques Crinon et Soizic Pachet, comporte une bibliothèque hypertextuelle contenant environ deux cent cinquante extraits de romans d'expérience personnelle que l'apprenti scripteur peut consulter, soit par l'intermédiaire d'une liste de critères, soit, de manière plus libre, par des liens hypertextuels [CRI 97]. Conçu autour du même principe, LYRE d'Éric Bruillard, est un système d'aide à l'analyse de textes littéraires en vue de la rédaction d'un commentaire composé :

«Sur un poème ou un texte littéraire, LYRE offre divers points de vue de lecture (lexique, images, niveaux phonique et prosodique, syntaxe, sémantique), des possibilités de croiser ces divers points de vue en les visualisant sur le texte initial et d'effectuer une recherche transversale à l'aide d'un point de vue global » [BRU 96 : 262].

D'ambition plus modeste, des expériences ponctuelles ont également été menées à l'initiative d'enseignants de l'enseignement secondaire ou supérieur². On peut citer l'exemple de cette petite application réalisée par Claudine Dubois destinée à exercer les élèves à la lecture méthodique [DUB 98] et dont le système d'aide éclaire certains aspects du texte.

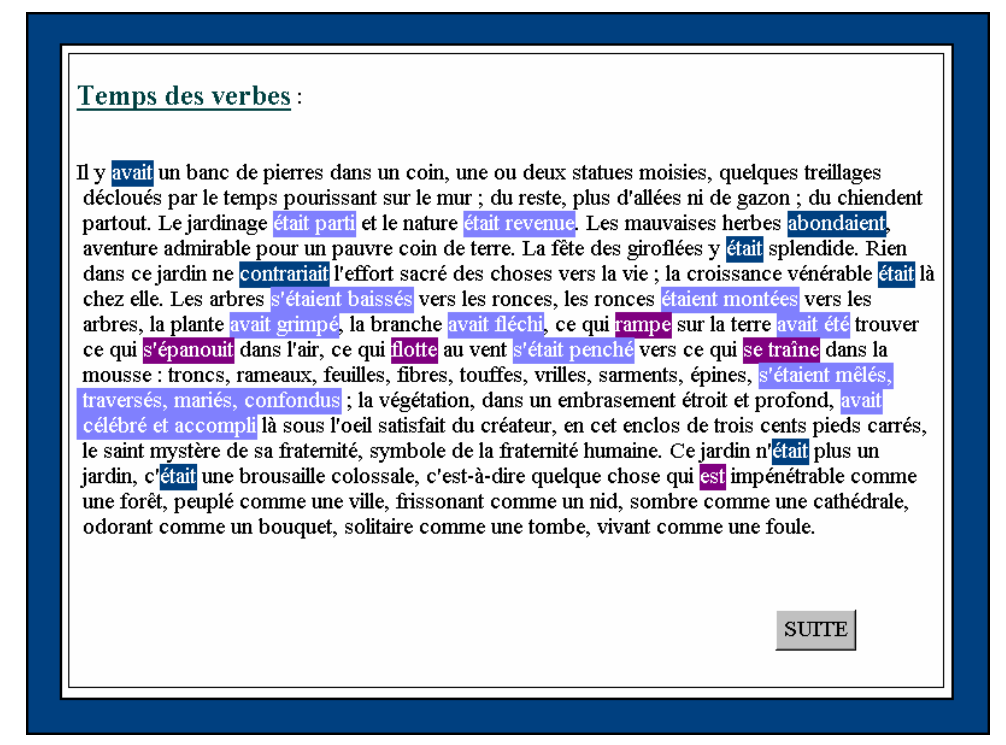

Figure 1. Page d'aide correspondant à la rubrique «temps des verbes» (extrait des Misérables, description du jardin de Cosette, IVe partie, livre III, chapitre 3)

\footnotetext{
${ }^{2}$ Voir [BES 93].
} 
Pourtant, l'informatique n'est jamais mise au service d'une réflexion consciente sur la nature de la lecture littéraire. Pour cela, il faudrait non seulement qu'elle s'inscrive dans le cadre d'une didactique de la lecture mais aussi que ce soit à l'élève d'élaborer son propre hyperdocument à partir d'un texte imprimé, c'est-à-dire de proposer sa lecture d'un texte, d'exposer son propre métatextuel. Il ne faut pas non plus perdre de vue que «pour qu'il y ait une réelle appropriation du savoir, tout apprentissage doit être associé à une pratique de production et de recherche » [ORI $86: 5]$.

Il ne s'agirait donc pas là d'une écrilecture au sens où l'entendent Alain Vuillemin et Arnaud Gillot, c'est-à-dire d'une interaction entre l'utilisateur d'un hypermédia et cet hypermédia, mais d'une écrilecture en quelque sorte factice, puisqu'il serait question, à seule fin éducative, de reproduire à l'écran un ensemble de comportements physiques (souligner, insérer des commentaires, avancer ou reculer de quelques pages) et d'opérations mentales (construction du sens).

\section{Vers une didactique de l'écrilecture}

En termes psycholinguistiques [COI 96], construire une représentation cognitive de ce qui est dit par le texte met en jeu à la fois des opérations concernant l'activation de réseaux d'unités de la mémoire à long terme, à partir des unités d'entrés (phonèmes, syllabes, mots), et des opérations d'intégration qui correspondent à la mise en relation des différentes unités ainsi qu'à leur organisation dans des unités plus globales. La grande quantité de nœuds spontanément activés lors de la première phase, dite bottom-up, est ensuite considérablement réduite lors de la deuxième phase qui désactive les éléments non pertinents. C'est ce qu'Umberto Eco appelle l'aimantation/narcotisation de propriétés sémantiques, l'identification du topic (le thème dans l'opposition thème/rhème) jouant selon lui un grand rôle dans l'actualisation des sèmes appropriés [ECO 85].

Par ailleurs, parce que tout texte comporte beaucoup d'informations implicites, un troisième type d'opération cognitive intervient concernant l'interprétation du message, à savoir l'intervention de connaissances usuelles, de schémas, relatifs tout aussi bien à l'organisation des textes qu'aux contenus, domaines et événements qui y sont évoqués. Pour comprendre la phrase : «Jean devait organiser un cocktail et il alla au supermarché », il faut activer deux scénarios, l'un «qui spécifie que, parmi les autres conditions sociales de réalisation d'un cocktail, il comporte la distribution de boissons, liqueurs et amuse-gueule », et l'autre qui prévoit que l'on vend dans les supermarchés, «entre autres articles, des boissons, des liqueurs et des amusegueule » (id.).

Si les logiciels d'apprentissage de la lecture constituent les seuls aides à la construction d'une représentation textuelle intégrée (Elsa de l'Association Française pour la Lecture ou Lirebel chez Chrysis par exemple), les dispositifs de lecture électronique peuvent en revanche se révéler un auxiliaire précieux pour mettre en 
relation les «blancs» du texte, les lieux d'indétermination, selon leur nature («blancs» relatifs à l'histoire, à la science, à la culture, etc.) [ISE 97], et en comblant le déficit d'informations explicites grâce à l'insertion d'annotations ou à des renvois vers une base de documents externes. La didactique de la lecture littéraire accorde une grande importance à cette phase qui renvoie à la fois, pour reprendre les termes d'Iser, aux textes antérieurs, mais également aux normes sociales et historiques, au contexte socioculturel au sens le plus large d'où le texte est issu. Cela se traduit en ce qui concerne le premier point, par une attention portée à la notion d'intertexte et, à propos du second point, à la morale, aux mythes, à l'interculturalité, à l'intermythologie, au stéréotype et au prototype ([TAU 96], [AMR 98], [FOU 00]), bref, à tout ce qui constitue «l'extra-esthétique » selon Iser.

Pour autant, lire n'implique pas seulement la mise en œuvre d'opérations cognitives mais aussi intellectuelles. Lorsque Umberto Eco se penche à la fin de son livre sur une nouvelle d'Alphonse Allais, la mise en évidence de l'écart entre une lecture naïve et une lecture critique du texte ${ }^{3}$ s'appuie sur l'analyse des stratégies discursives et narratives utilisées. C'est ainsi qu'il est amené à s'intéresser aux fonctionnements langagiers (épigraphes, formules de connivence, références à des scénarios communs et intertextuels, figures, etc.) qui trahissent le projet de l'auteur, ou tout du moins la complicité feinte du narrateur.

Cette attention portée au langage est la conséquence directe de l'autoréflexivité du discours de fiction. La littérature se sert de la fonction référentielle du langage (signes de choses) pour amener une réflexion sur le langage lui-même (signes de signes) et à travers elle une réflexion sur le monde. De la même façon que les paradigmes linguistiques (temps verbaux, reprises anaphoriques, etc.) garantissent la cohérence du texte, les paradigmes poétiques, par le retour régulé du même, en assurent la «coalescence» (à partir de la définition qu'en donne le Littré : « union des parties auparavant séparées... ») [ORI 88b]. La seule différence est la variation. Les paradigmes linguistiques ne suscitent aucune réaction de la part du lecteur car ils reviennent d'un texte à l'autre. Au contraire, les paradigmes poétiques, selon leur degré de créativité, attirent l'attention.

«Les mises ensemble qu'il faut effectuer pour produire du sens ne sont plus seulement celles que réclament les textes informatifs. Certes, comme pour ces derniers, il faut suivre l'organisation linéaire syntagmatique et être capable d'organiser les signifiés en propositions sémantiques qui créent l'effet de fiction à partir du travail de la vraisemblance. Mais il faut non moins apprendre à lire dans le translinéaire, les trouvailles de la ressemblance » [ORI 88a]

\footnotetext{
${ }^{3}$ La nouvelle d'Alphonse Allais, Un drame bien parisien, repose sur le procédé du malentendu. Après avoir laissé entendre une issue bien classique à son récit d'adultère, l'auteur parvient à plonger le lecteur dans la plus profonde perplexité, celui-ci ne sachant plus à quelles inférences interprétatives se fier...
} 
L'hypertexte pourrait là se révéler utile. Il peut permettre de visualiser les réseaux de relations qui existent entre les mots. Comment en effet mieux mettre en évidence l'inscription du paradigme dans le syntagme, ce qui, selon Jakobson, caractérise la fonction poétique? Une telle utilisation s'intégrerait d'ailleurs parfaitement dans le cadre d'une didactique de la lecture littéraire qui prend en compte l'autotélisme du texte (lecture ascendante) et qui, par le rétablissement des informations implicites, s'intéresse également au système de référence de l'élèvelecteur (lecture descendante).

Elaborer un hyperdocument lectoral, dans le but de donner à l'écran une représentation de la lecture, reviendrait en définitive à deux principales opérations. Insérer des annotations visant à expliquer, reformuler, contextualiser, documenter, etc., de façon à relier des passages du texte à tout ce qui constitue l'extratextuel et relier entre eux les éléments internes au texte qui se ressemblent et fondent ainsi la poéticité. 


\section{Récit d'une expérience}

J'ai eu l'occasion pendant l'année scolaire 1999/2000 d'expérimenter ce modèle didactique avec des élèves de CM2 de l'école annexe Charles Daviler de Montpellier, en collaboration étroite avec leur instituteur, Claude Veau, également formateur à l'IUFM.

Le choix du texte de départ s'est porté sur une nouvelle extraite d'Usurpation d'identité de Boileau-Narcejac, intitulée Le Gorille aux Floralies [BOI 80]. Écrite à la manière de Dominique Ponchardier, auteur de romans d'espionnage, lui-même ancien agent des services secrets, elle met en scène le héros de la série des Gorille aux prises avec ses homologues étrangers à propos d'un accumulateur miniaturisé (voir annexe).

Si nous nous sommes très rapidement tournés vers le genre policier, c'est principalement en raison de la place privilégiée qu'occupe le suspense dans la motivation des enfants pour la lecture. Il présente en outre l'avantage d'être une métaphore de l'activité de lecture. Ce n'est certainement pas sans raison que les deux analyses textuelles de Barthes prennent successivement pour objet deux récits à énigme.

Le choix du genre espionnage n'a en revanche rien de réfléchi. Il se trouve que les nouvelles proposées en librairie et destinées à des enfants de 10-11 ans sont rares, celles-ci s'adressant plutôt à une classe d'âge inférieure. En témoignent les sujets des histoires proposées qui mettent rarement en scène de vrais délits mais tournent plus volontiers autour de vols de chewing-gum ou de crayons. C'est en cherchant dans la littérature adulte, après avoir écarté bon nombre de nouvelles noires susceptibles de heurter la sensibilité des enfants, que nous avons choisi cette nouvelle à la fois pour sa longueur (sept pages), l'originalité de son monde de référence et son ton humoristique.

La transformation de la nouvelle du Gorille aux Floralies sous forme d'hyperdocument s'est étalée sur toute une année scolaire, à raison d'une séance d'une heure et demie par semaine environ. D'une façon générale, pour chaque étape de la réalisation, le travail s'effectuait tout d'abord individuellement puis, après une première confrontation des résultats de chacun à l'intérieur de sous-groupes, une phase de mise en commun permettait d'aboutir à une synthèse.

Pendant l'expérience, mon rôle s'est tenu à celui de conseiller, de consultant auprès de l'enseignant. Je veillais à ce que les consignes de travail, les explications, et d'une manière générale la conduite du cours restent toujours au plus près des objectifs didactiques fixés. J'ai en de rares occasions pris la parole et mené des activités, et cela à chaque fois qu'un point théorique posait problème, comme la notion de fonction par exemple. 


\section{Démarche}

\subsection{Lecture du texte}

La première étape a été consacrée à la lecture du texte. La mise en scène didactique pour laquelle nous avons optée a consisté à faire découvrir progressivement la nouvelle aux élèves. Ceci a permis de faire naître les premières hypothèses de sens.

Le Gorille aux Floralies est en effet une nouvelle assez singulière, puisque tout en présentant de fortes homologies avec les caractéristiques du genre de l'espionnage, elle s'en éloigne cependant par différents aspects. D'une manière générale, et le recours à un lexique familier, populaire et argotique y est pour beaucoup ${ }^{4}$, le monde de l'espionnage décrit dans la nouvelle de Boileau-Narcejac est moins policé, moins raffiné que celui dépeint traditionnellement dans les romans appartenant au même genre. Ce qui diffère des autres récits d'espionnage n'est pas le thème de l'intrigue - qui pourrait se formuler ainsi: «Défense du potentiel économique et militaire/Contre le communisme/dans un pays d'Occident ou du Tiers Monde », et que l'on retrouve selon Erik Neveu dans 25\% des romans d'espionnage [NEV 85] -, ni non plus sa structure, mais l'atmosphère. C'est une partie du monde de référence qui change, un monde où il est admis de prendre quelque liberté avec certaines conduites sociales, tant du point de vue du comportement que de celui de la langue. Un monde où les hommes ne sont pas des superhéros, mais des êtres ordinaires, peut-être même par moments «inférieurs », au sens où Aristote emploie ce terme à propos de la comédie, au commun des mortels, mais suffisamment hors normes pour justifier leur appartenance à « l'élite » de la nation.

La peinture d'un monde à la française, avec son franc-parler (à travers un certain niveau de langue on l'a vu, mais aussi par le recours régulier à l'hyperbole et à l'ironie) et son irrespect des conventions, dont le cinéma a d'ailleurs exploité la veine pendant quelques années ${ }^{5}$, conduit inévitablement à une désacralisation, à une démythification du milieu de l'espionnage. Démythification qui par contrecoup

\footnotetext{
${ }^{4}$ Il est effectivement impossible de disjoindre totalement la valeur stylistique d'un terme de sa valeur péjorative ou méliorative. «La valeur (connotative) attachée au signifiant linguistique et la valeur (dénotative) attachée au référent ont tendance à s'influencer mutuellement. C'està-dire qu'un terme connoté "vulgaire » a tendance, par une sorte d'effet de contagion, à vulgariser le dénoté auquel il renvoie; et, inversement, les termes stylistiquement «normaux » qui désignent des réalités sexuelles ou scatologiques ont tendance à être perçus comme «bas » dans la mesure où la dévalorisation qui s'attache au départ au seul dénoté finit par rejaillir sur le mot lui-même » [KER $77: 101]$.

${ }^{5}$ Le Gorille a été adapté plusieurs fois au cinéma avec Lino Ventura (Le Gorille vous salue bien de Bernard Borderie, 1958) puis Roger Hanin dans le rôle titre (La valse du Gorille de Bernard Borderie, 1959, Le Gorille a mordu l'archevêque, Maurice Labro, 1962).
} 
laisse apparaître toute la dérision de la condition de l'espion qui va jusqu'à se matérialiser parfois dans des situations véritablement cocasses ${ }^{6}$.

Pour une personne maitrisant l'ensemble de ces connaissances, Le Gorille aux Floralies n'apparaît que comme un avatar d'un genre hypercodé que l'on pourrait qualifier d'espionnage à la française. Elle n'éprouve aucun mal à combler les innombrables blancs du récit. Cela n'a pas été le cas pour les élèves de CM2. La plupart des références historiques et architextuelles (relative au genre de l'espionnage) leur manquaient et c'est la découverte par fragments du texte qui a permis aux élèves, par la médiation de l'enseignant, de combler leurs lacunes et d'émettre des hypothèses.

\subsection{Fragmentation du texte}

La deuxième étape a consisté à découper le texte en pages-écran ou unités d'information. Les enfants ont pour ce faire recouru à des critères aussi variés que le changement de lieu ou de personnages, le passage d'un type d'action à un autre, le suspens ou encore le «sujet». Le nombre de parties variait d'un élève à l'autre de 7 à 14 .

Nous leur avons par la suite fourni un cadre épistémologique construit sur la base d'une synthèse de la logique décisionnelle de Brémond, porteuse d'une définition claire des notions de fonction (tout action ou événement peut être appréhendé en trois temps : avant/pendant/après), de séquence élémentaire et de leur combinaison, et du schéma narratif de Greimas qui «constitue comme un cadre formel où vient s'inscrire le «sens de la vie »...» [GRE 93]. A partir de leur découpage mais aussi après une nouvelle lecture du texte, les élèves, par groupes, ont donc cherché à regrouper par paires les événements qui selon eux ouvrent et ferment les différentes séquences du texte. Par exemple : début de la mission / fin de la mission; Geo n'a pas de plan / Geo a un plan; Geo ne comprend pas les paroles de Kauffman / il les comprend, etc. Il ne restait plus ensuite qu'à faire l'inventaire des actions intermédiaires permettant de passer de l'état initial à l'état final de chaque séquence.

Ce travail a débouché sur la réalisation d'un tableau à double entrée, chaque colonne représentant une séquence (par exemple: recherche d'un plan, compréhension des paroles de Kauffman) et chaque ligne une fonction (recherche dans les fichiers / échec / recherche d'une autre idée). Ce sont ces fonctions qui constituent les 33 pages-écrans de l'hyperdocument lectoral.

Cette étape a permis une première approche de la poéticité des récits en sensibilisant les enfants au retour réglé, dans le syntagme, d'éléments issus de paradigmes narratifs [JAK 63]. La nouvelle de Boileau-Narcejac appartenant au genre policier, ce principe poétique est mis au service du suspense. Dans la quête du

\footnotetext{
${ }^{6}$ Voir pour une analyse plus en détaillée de la nouvelle [SOU 01].
} 
héros, les énoncés d'état qui suivent des énoncés de faire ne correspondent pas toujours à une conjonction à l'objet. Ils peuvent équivaloir à une nouvelle disjonction (le héros est sur le point d'arraisonner le voleur, mais ce dernier se révèle meilleur coureur...) ou encore à un état non encore stabilisé, indécis. Deux quêtes, par ailleurs, l'une pragmatique (récupérer un objet volé), l'autre cognitive (résoudre une énigme), coexistent au sein du récit, la résolution de celle-ci retardant l'aboutissement de celle-là. En détournant une formule de Barthes, on pourrait dire en somme que «de même que la rime (notamment) structure le poème selon l'attente et le désir du retour, de même les codes herméneutiques [et proaïrétiques] structurent l'énigme [et la diégèse] selon l'attente et le désir de sa résolution [de leur achèvement] $»[$ BAR 70].

C'est d'ailleurs pour mieux leur faire prendre conscience de ce retour de l'énigme que nous leur avons demandé de marquer les lieux où elle «se centre, se pose, se formule, puis se retarde et enfin se dévoile » [BAR $70: 23]$.

\section{3 création de liens hypertextuels}

Une fois ce travail effectué sur la macrostructure, il a ensuite fallu se pencher sur les microstructures. On a vu que les élèves avaient déjà eu l'occasion, grâce à la découverte progressive du texte, de se confronter à certaines subtilités. Mais cette première lecture leur a aussi permis d'apprécier l'humour de la nouvelle, perceptible non seulement à travers le lexique populaire, familier et argotique, mais également par la cocasserie de certaines situations et par certains aspects du Gorille, irrespectueux des convenances et adoptant bien souvent un regard ironique sur sa mission.

Après avoir convenu ensemble, à l'issue d'une première phase de travail et de mise en commun, que l'humour du texte résidait essentiellement dans le choix du lexique, l'emploi de l'hyperbole, et les emprunts, par le Gorille, à d'autres sociolectes (publicité, armée, religion, etc.), les élèves ont eu pour tâche de repérer dans le texte, au moyen de photocopies et de surligneurs, les passages qui relevaient de tel ou tel paradigme.

Pour symboliser la métamorphose progressive du texte, des feuilles de papier A4, des fils de laine de différentes couleurs et un marquage au feutre matérialisaient sur un mur de la classe les unités d'information, les liens hypertextuels et les ancres. Chaque nouvelle séance voyait la trame se tisser de nouveaux fils.

Au fur et à mesure de l'avancée du projet, je me suis chargé du versant informatique, c'est-à-dire de la réalisation de l'hypertexte électronique : numérisation de la nouvelle, découpage, élaboration de l'architecture, création des pages-écran et des liens, conception de la palette graphique, recherche d'une navigation optimale, 
etc $^{7}$. A quatre reprises, les élèves ont été initiés à certaines opérations d'édition dans une salle informatique de l'IUFM. Ils ont ainsi appris à créer des «tiroirs » c'est-àdire, dans la terminologie du logiciel de génération d'hypertextes que nous avons utilisé, des fenêtres, à écrire ou importer un texte de fond ou une image, à créer des hypermots, des «efficaces» (liens), à poser un bouton, à le déplacer et à le redimensionner, à changer les couleurs de fond d'écran et de fond de tiroir, etc. A l'issue de chacune de ces séances, la version de l'hypertexte qui correspondait à l'état des travaux du moment leur était présentée. Ils pouvaient ainsi se rendre compte de l'avancée du projet et surtout donner du sens aux différentes activités d'analyse textuelle qui leur étaient proposées.

\footnotetext{
${ }^{7}$ Logiciel utilisé : Polygraphe 5, développé par Jean-Marie Ball. Une version « light » est disponible gratuitement à l'adresse : http://home.nordnet.fr/ jmball/accueil.html.
} 


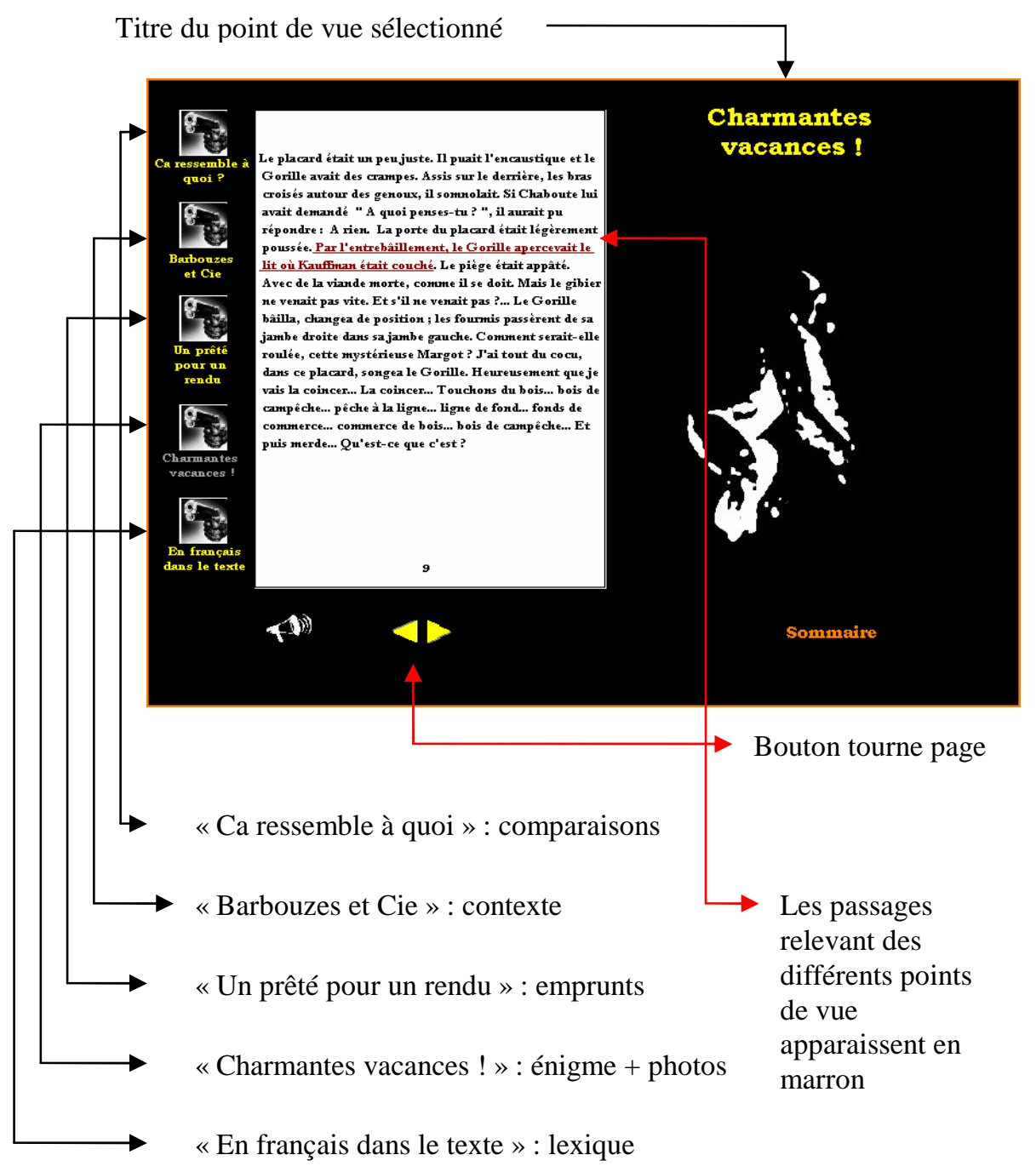

Figure 2. Description d'une page-écran type du cédérom

\subsection{Ajout d'annotations}

La dernière étape a consisté dans l'ajout d'annotations. Un grand nombre d'entre elles, touchant au genre de l'espionnage, au contexte socio-historique de la nouvelle et d'une façon générale à la série des Gorille, ont été plus ou moins écrites au 
moment de la première lecture du texte, lorsqu'il s'agissait justement de rassembler les connaissances encyclopédiques nécessaires à la compréhension du texte (extratexte). Ces ajouts correspondent dans la plupart des cas, selon la typologie des actes annotatifs élaborée par une équipe de chercheurs de l'Institut de recherche en Informatique de Toulouse [MAZ 95] à des annotations destinées à contextualiser certains passages.

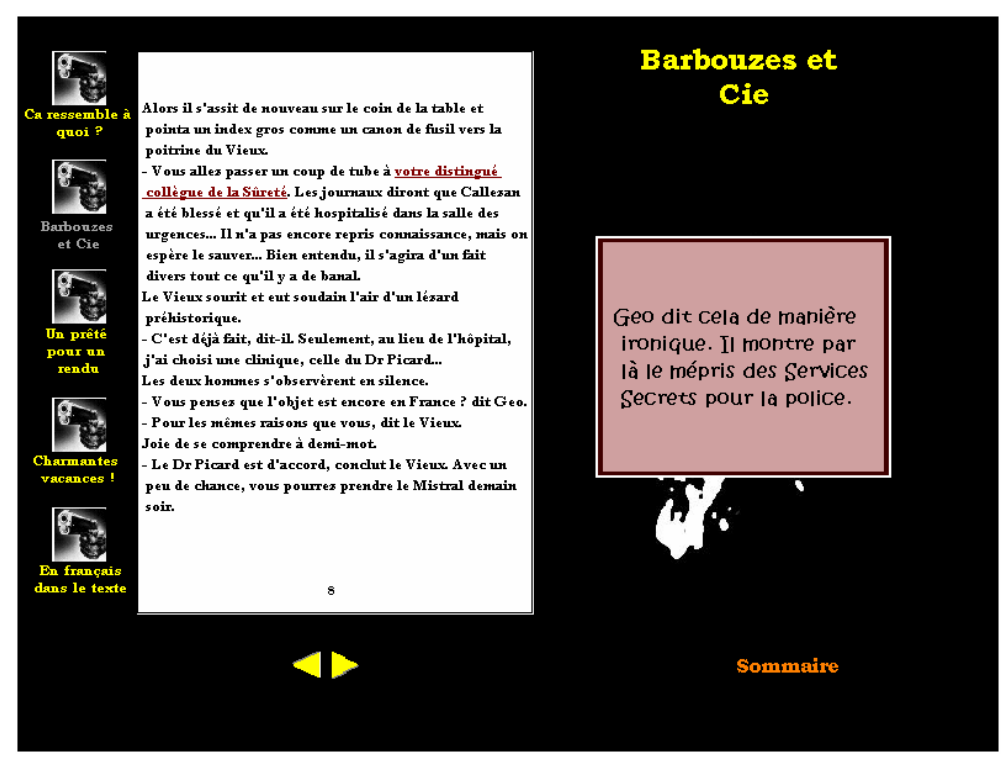

Figure 3. Exemple d'annotation relative au contexte

Les autres annotations ont été écrites au cours de cette phase. Celles relatives au lexique n'ont pas fait l'objet de consignes particulières de notre part et sont à classer dans les commentaires du type «attirer l'attention». Pour les comparaisons en revanche (intratexte), nous avions donné comme instructions aux élèves, non seulement de commenter celles qu'ils trouvaient les plus cocasses, les plus drôles, mais également d'en écrire à leur tour sur les différents modèles que compte la nouvelle (rapport d'analogie le plus extravagant possible expliqué soit par une conjonction, soit par un système de comparaison quantitative, soit par un verbe à sens comparatif, soit par un substantif non actualisé). Cette consigne était en fait dictée par notre souci de lier lecture et écriture littéraire. 


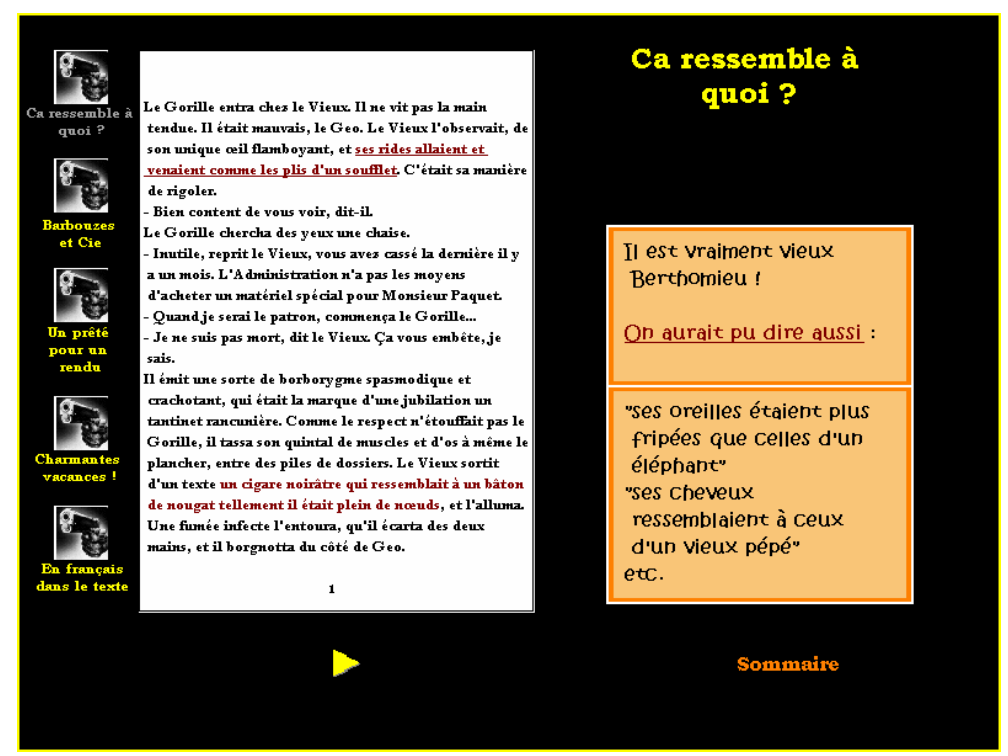

Figure 4. Exemple d'annotation relative aux comparaisons. Les comparaisons écrites par les élèves "à la manière de » apparaissent à l'écran après un clic de souris sur «On aurait pu dire aussi »

L'énigme a elle fait l'objet de la part des élèves d'un traitement particulier. Sensibilisés par plusieurs séances de travail sur la progression de l'énigme, dont chacune de ses manifestations a pour seul objectif d'emmener le lecteur sur une fausse piste, de le leurrer, ils ont non seulement tenu à ce que leur commentaires ne dévoilent pas l'énigme mais, bien au contraire, la renforcent. Par exemple, lorsque le Vieux rapporte les dernières paroles de Kauffman ( «La batterie... Marguerite... Marguerite »), le commentaire qui apparaît à gauche de l'écran est : « le voleur est donc une voleuse », hypothèse que le lecteur et le héros sont amenés à formuler (Marguerite est un prénom féminin), alors qu'en réalité «marguerite » désigne une fleur et que le voleur n'est autre qu'un homme.

Autre artifice. A l'écran, le lien n'apparaît pas en caractères de couleur. L'utilisateur doit donc, pour afficher le commentaire, déplacer sa souris, au hasard ou non, dans le corps du texte jusqu'à l'apparition du cadre jaune. Il s'agit encore une fois de préserver l'intégrité de l'énigme qui, par définition, est cachée. 


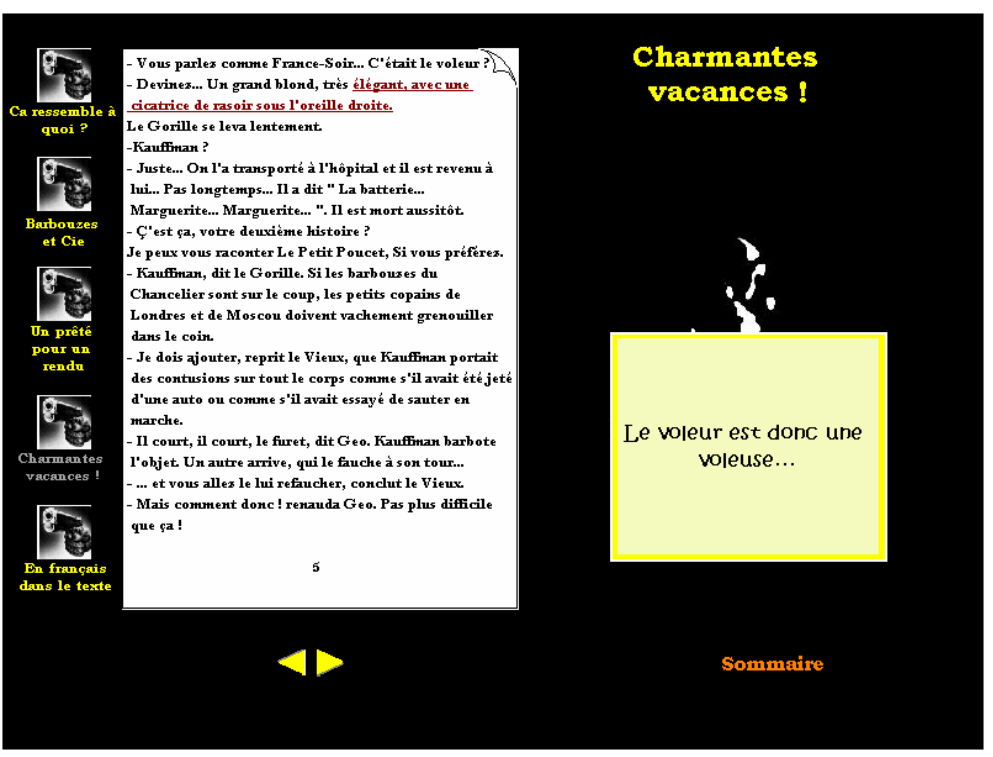

Figure 5. Exemple d'annotation relative à l'énigme

Un des procédés humoristiques du texte a également fait l'objet d'une mise en scène analogue. Il s'agit des emprunts, qui parsèment le discours du Gorille, à d'autres discours appartenant à des sphères professionnelles bien identifiées : l'armée, l'administration, le journalisme, etc. (intertexte). A l'image de cette scène où, planqué dans le placard d'une chambre d'hôpital, tandis que le cadavre qu'il surveille «floflope », le Gorille exprime toute la dérision de sa situation sur le mode du slogan publicitaire : «Charmantes vacances! Vue imprenable, odeurs sylvestres. Un petit coin bien peinard ». De façon à bien mettre en évidence le procédé, nous avons essayé, à chaque fois que cela était possible, de reconstituer le contexte original supposé de l'expression. Ainsi cet autre exemple où l'ironie du Vieux à l'égard de la police («Votre distingué collègue de la Sûreté ») est signalée par la superposition à l'écran d'une lettre officielle (drapeaux, signature du Président de la République, tournures) $)^{8}$.

\footnotetext{
${ }^{8}$ Je tiens à souligner que si l'idée de reconstituer le contexte d'origine de certains passages vient de nous, les documents (lettre, publicité, enregistrement audio) ont été réalisés par les élèves.
} 


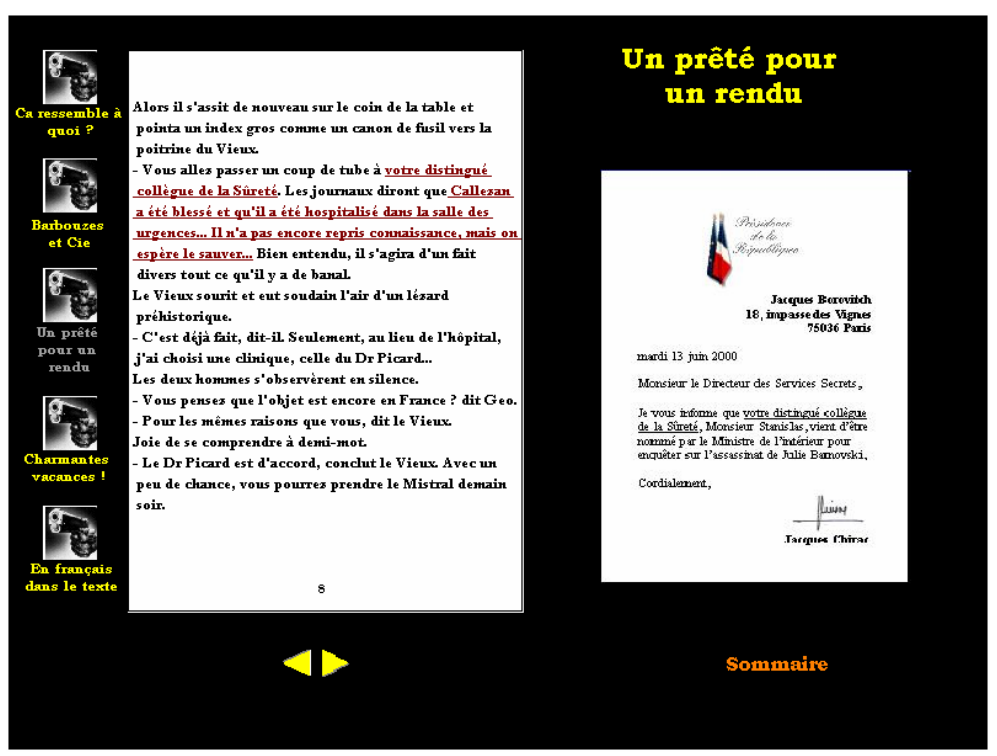

Figure 6. Exemple d'annotation relative aux emprunts (intertexte). L'extrait recontextualisé du texte est souligné dans la lettre.

On voit comment les choix scénaristiques que nous avons été amenés à faire ont été guidés par le souci de représenter de la façon la plus significative possible les rapports existants à l'intérieur du texte (intratexte) et entre le texte et l'extérieur du texte (extratexte et intertexte).

Bien qu'il ne s'agisse pas d'annotations, d'autres ajouts ont été apportés au texte d'origine. Dans Le Gorille aux Floralies, certains monologues du héros sont au discours indirect libre. Si bien qu'il est parfois difficile pour le lecteur d'identifier l'origine de la voix. Qui parle ? Le héros ? Le narrateur? L'auteur ?9 La perte de l'origine des voix est selon Barthes une des caractéristiques du texte « ouvert » qui permet au lecteur de coopérer avec lui, d'interagir. Ces passages ont fait l'objet d'enregistrements par les élèves. Ils sont signalés par l'icône d'un porte-voix en bas à gauche de l'écran (voir figure 1). Cliquer dessus ouvre le fichier son et fait apparaître le passage concerné en bleu.

Enfin, pour laisser une certaine marge de liberté aux enfants, nous avons accepté qu'ils parsèment le texte de photos. La seule condition était qu'elles reprennent des descriptions (propositions relatives, adverbes, adjectifs, séquences). Cela leur a permis de prendre conscience que les descriptions sont loin d'être toutes assimilables

9 Rappelons que l'auteur imité par Boileau-Narcejac s'appelle Dominique Ponchardier, ancien agent des services secrets et dont on imagine sans peine que son langage était proche de celui de son héros. 
à des images, qu'elles peuvent aussi, par exemple, apporter des informations sur les comportements des personnages, sur leurs relations, etc.

\section{Conclusion et perspectives}

\section{$5.1 \quad$ Un bilan positif}

On peut raisonnablement tirer de cette expérience un bilan positif. $\mathrm{La}$ transformation du Gorille en hypertexte a bien permis de mettre à plat les opérations cognitives et intellectuelles en œuvre lors de la lecture. Qu'est-ce que lire en effet, sinon construire, comme nous l'avons fait, un réseau de renvois internes et associer des passages à d'autres données ?

«Lire un texte, c'est retrouver les gestes textiles qui lui ont donné son nom [...]. C'est aussi [rapporter] le texte à d'autres textes, à d'autres discours, à des images, à des affects, à toute l'immense réserve fluctuante de désirs et de signes qui nous constitue » [LEV].

L'objet de ce travail était de le démontrer théoriquement, de préciser le contexte sociologique de ce rapprochement entre une technologie intellectuelle et une activité mentale (évolution des pratiques de lecture/écriture) et d'établir, dans le cadre d'une certaine tradition de l'utilisation de l'informatique à l'école, qu'il était possible d'exploiter en classe cette analogie.

\subsection{Un point faible : l'évaluation}

Quant à savoir si les enfants ont développé, au cours de ce travail, des compétences relatives à la lecture de textes littéraires, il m'est difficile, sinon impossible, de répondre précisément à cette question. Ce qui comptait avant tout était de prouver la faisabilité de l'hypertextualisation d'un texte en classe et de délimiter les enjeux propres à ce genre d'activité. Mettre en place un dispositif évaluatif aurait nécessité de définir préalablement, et ce de façon précise, un référentiel de compétences, de telle sorte qu'il soit possible de comparer les données issues d'un test diagnostic établi en début d'année avec celles recueillies à la fin de l'expérience. Or, l'état d'avancée de mes recherches au moment de l'expérience ne m'aurait pas permis de fixer de manière suffisamment claire la liste des objectifs pédagogiques à atteindre. Le rôle par exemple que pouvait jouer l'hypertexte dans la levée des lieux d'indétermination du texte ne s'est révélé une préoccupation qu'en cours de projet, une fois confronté aux difficultés de compréhension des élèves. L'analyse de la nouvelle, d'autre part, n'avait pas encore son caractère définitif et aucune typologie de liens hypertextuels n'était disponible. Vérifier la portée cognitive de ce type de projet aurait nécessité de mettre en place, après avoir analysé 
en détail notre procédure et en avoir déduit les forces et les faiblesses, une seconde expérience intégrant les principes d'une évaluation.

\subsection{Vers la conception d'un logiciel d'écrilecture}

La lourdeur de la réalisation informatique a en revanche mis en évidence la nécessité de pouvoir disposer d'un logiciel qui facilite certaines opérations d'édition. Le système SEVE par exemple, qui a permis à Éric Bruillard de réaliser diverses applications dont LYRE, aide à la rédaction d'un commentaire composé, présente, entre autres, les caractéristiques suivantes :

- « un système hypertexte et un système de fenêtres, des ancres, visualisées par des surbrillances, pouvant être associées à des entités (mots ou groupes de mots) et des champs ;

- des points de vue associés à l'hypertexte (attributs de liens), permettant de changer les explications et de transformer dynamiquement le document par la visualisation des ancres [...] » [BRU 96 : 260].

Qu'il soit possible de typer les liens est déjà un net progrès, car il faut dans le cas contraire - il en fut ainsi pour Le Gorille aux Floralies - attribuer une couleur à chaque hypermot, tâche relativement fastidieuse. Mais il reste le problème des points de vue. Créer des liens de différentes couleurs est une chose, faire en sorte qu'ils ne se superposent pas en est une autre. L'idée en effet n'est pas d'associer à une pageécran plusieurs points de vue ${ }^{10}$ mais un seul. A l'heure actuelle, aucun logiciel, à ma connaissance tout du moins, ne propose cette fonctionnalité. La solution consiste alors à dupliquer le texte autant de fois qu'il existe de points de vue. C'est ainsi que le cédérom du Gorille, qui à priori se présente comme une succession de 33 pagesécran, en comprend en réalité environ 198 (soit 33 pages multipliées par le nombre d'entrées: comparaisons, contexte, intertexte, descriptions, lexique et discours indirect libre $\left.{ }^{11}\right)$.

Il faudrait également que l'insertion d'annotations et de commentaires soit simplifiée, qu'il ne soit pas nécessaire de définir les couleurs de fond, de fenêtre et de cadre et qu'un emplacement spécial à l'écran leur soit réservé. Il serait nécessaire en un mot d'automatiser les différentes tâches impliquées dans l'édition hypertextuelle d'un texte.

\footnotetext{
${ }^{10}$ Quoique cela puisse correspondre à un mode de consultation possible. Dans LYRE, un point de vue «Global» permet de croiser les différents points de vue en les visualisant sur le texte initial. "Toutes les expressions expliquées suivant un point de vue quelconque sont mises en surbrillance. En cliquant sur un mot [...], on peut choisir l'explication liée à un point de vue particulier » [BRU $96: 263$ ].

${ }^{11}$ Descriptions et énigme, bien qu'étant deux points de vue différents, sont visibles à partir d'un seul et même bouton.
} 
Peut-être même ne faudrait-il pas s'en contenter. Si la mise au point d'un tel logiciel devait avoir lieu, pourquoi alors ne pas en profiter pour intégrer certaines fonctionnalités lexicométriques ? Cela complèterait avantageusement un dispositif de lecture déjà performant ${ }^{12}$. Pour Alain Vuillemin en effet, une utilisation réellement créative de l'informatique consiste à lier fonctions hypertextuelles, recherche et gestion d'annotations [VUI 99]. Des dispositifs de ce type sont mis à la disposition des chercheurs à la Bibliothèque nationale de France. Véritables stations de travail sur PC, ces postes de lecture assistée par ordinateur (PLAO) sont équipés de toute une panoplie d'outils pour charger et héberger les textes sélectionnés, pour naviguer en mode image dans des textes qu'il est loisible de repérer, surligner, souligner, annoter, comparer grâce au multifenêtrage, et dont il est possible d'extraire des souscorpus.

Pourquoi ne pas adapter ces pratiques à l'école ? Il n'est bien sûr pas question qu'elles se substituent à celles en vigueur, ni non plus d'ailleurs qu'un dispositif électronique vienne remplacer les supports traditionnels de cours (quoique certains y aient déjà pensé ${ }^{13}$ ), mais de mettre à la disposition des élèves un environnement d'apprentissage ouvert, riche et stimulant qui fasse évoluer leur rapport au texte littéraire et à la lecture. C'est dans ce cadre plus large que devrait en définitive s'insérer l'hypertexte lectoral si son usage devait se généraliser.

Un tel dispositif d'aide à la lecture, qui prendrait place à côté de logiciels d'aide à l'écriture qui existent déjà en grand nombre, comme L'auteur en herbe, Gamme d'écriture, L'atelier d'écriture (CDE4) reconnu d'intérêt pédagogique par le MEN), ou encore GramR Junior dans le domaine de l'aide à la relecture/révision, serait le premier du genre. Si l'on écarte en effet les produits qui visent plus simplement à «développer le goût de la lecture » (Le Petit Prince de Jériko) et ceux dont le contenu tient essentiellement en des activités de soutien et de remédiation centrées sur la compréhension de l'écrit, concevoir un logiciel simple d'utilisation qui permette de manipuler des textes (interrogation, annotations, insertions de liens) et, qui plus est, offre la possibilité d'approcher la nature intime de la lecture et la spécificité littéraire témoigne d'une toute autre ambition. On quitte là le domaine de l'EIAO et des environnements interactifs (type LYRE ou Gamme d'écriture) pour celui de l'expérimentation et de l'exploration des connaissances.

\footnotetext{
12 Certaines techniques lexicométriques (conclusions d'ordre stylistique ou thématique qui peuvent par exemple être tirées à partir de listes de formes hors ou en contexte) peuvent facilement être au mises au service de la recherche de paradigmes poétiques.

${ }^{13}$ Le ministère de l'Éducation nationale a signé une convention avec Havas Éducation pour mener, à partir de la Toussaint 2000, une expérience dans cinq classes pilotes de troisième où chaque élève dispose d'un cartable électronique. Contenant pour l'heure des programmes d'histoire, de géographie et de sciences de la vie et de la terre, il pourra intégrer plus tard d'autres matières, ainsi que du son, des images et de la vidéo. Il serait question qu'il remplace à terme les manuels scolaires, si tout du moins ils font preuve de la même résistance que les livres!
} 


\section{Annexe : Le Gorille aux Floralies de Pierre Boileau et Thomas Narcejac [BOI 80]}

\section{A la manière de A. L. Dominique.}

Le Gorille entra chez le Vieux. Il ne vit pas la main tendue. Il était mauvais, le Geo. Le Vieux l'observait, de son unique œil flamboyant, et ses rides allaient et venaient comme les plis d'un soufflet. C'était sa manière de rigoler.

- Bien content de vous voir, dit-il.

Le Gorille chercha des yeux une chaise.

- Inutile, reprit le Vieux, vous avez cassé la dernière il y a un mois. L'Administration n'a pas les moyens d'acheter un matériel spécial pour Monsieur Paquet.

- Quand je serai le patron, commença le Gorille...

- je ne suis pas mort, dit le Vieux. Ça vous embête, je sais.

Il émit une sorte de borborygme spasmodique et crachotant, qui était la marque d'une jubilation un tantinet rancunière. Comme le respect n'étouffait pas le Gorille, il tassa son quintal de muscles et d'os à même le plancher, entre des piles de dossiers. Le Vieux sortit d'un tiroir un cigare noirâtre qui ressemblait à un bâton de nougat tellement il était plein de nœuds, et l'alluma. Une fumée infecte l'entoura, qu'il écarta des deux mains, et il borgnotta du côté de Geo.

- J'ai deux histoires à vous raconter.

- C'est deux de trop, fit le Gorille. Parce que je dois vous rappeler que je suis en congé régulier et que j'ai l'intention d'aller faire trempette du côté d'Antibes. Alors, vos histoires, vous pouvez vous les mettre où je pense.

Berthomieu n'avait pas toujours été le chef des barbouzes. Il savait, lui aussi, ce que représentait un congé de huit jours entre deux missions, quand on essayait d'oublier l'une et de ne pas penser à l'autre. Mais il adorait les rognes du Gorille.

- Vous n'êtes plus en congé, dit-il... Depuis une heure... Exactement, depuis qu'on m'a raconté la première histoire, et je l'ai écoutée, moi !

Le Gorille, avec une légèreté déconcertante, se trouva debout, la tête droite, les yeux fixés à quinze pas et le petit doigt sur la couture du pantalon.

- A vos ordres, grand-père.

Le Vieux sortit sa grimace $n^{\circ} 1$ et, un rien condescendant, répliqua :

- Repos, mon ami, repos.

Ils se regardèrent une minute, fignolant la comédie, en mettant juste un peu trop, l'un ravagé de tics et l'autre plus raide qu'un mannequin, puis Geo lâcha la pose, appuya sur le coin du bureau une fesse large comme une presse à emboutir et murmura gentiment :

- Si vous cessiez de déconner, croyez pas qu'on irait plus vite ?

Le dentier du Vieux faillit se débiner, il n'avait plus du tout envie de rigoler. Il consulta un papier qui traînait près de lui, dans ce qu'il appelait le foutoir, et qui 
était la partie du bureau où échouaient des cure-dents, des élastiques, des trombones, des douilles et jusqu'à des rognures d'ongles.

- On a cambriolé les Usines Weber, attaqua-t-il.

- Et alors, grommela Geo, on n'est pas des poulets.

- Chez Weber, on travaillait depuis trois ans à mettre au point un accumulateur extra-léger et les recherches venaient d'aboutir. Vous comprenez ce que ça veut dire, un accumulateur extra-léger?

- Vous fatiguez pas... Moi aussi, je lis Tintin. Continuez. On a fauché des plans?

- Non. On a enlevé un élément de batterie.

- Aussi sec ? Y avait même pas un rombier chargé des services de nuit ?

- Si. Il a été assommé.

- C'est gros, ce machin qu'ils ont barboté ?

- Un mètre trente-cinq de haut, trente kilos.

- Pour de l'extra-léger, ça se défend.

- Ça c'est la première histoire. Le vol a eu lieu vers 3 heures du matin. A 6 heures, deux agents cyclistes ont découvert, boulevard Masséna, un homme grièvement blessé ; une balle dans le foie.

- Vous parlez comme France-Soir... C'était le voleur?

- Devinez... Un grand blond, très élégant, avec une cicatrice de rasoir sous l'oreille droite.

Le Gorille se leva lentement.

-Kauffman?

- Juste... On l'a transporté à l'hôpital et il est revenu à lui... Pas longtemps... Il a dit «La batterie... Marguerite... Marguerite... ». Il est mort aussitôt.

- Ç'est ça, votre deuxième histoire ?

Je peux vous raconter Le Petit Poucet, Si vous préférez.

- Kauffman, dit le Gorille. Si les barbouzes du Chancelier ${ }^{1}$ sont sur le coup, les petits copains de Londres et de Moscou doivent vachement grenouiller dans le coin.

- Je dois ajouter, reprit le Vieux, que Kauffman portait des contusions sur tout le corps comme s'il avait été jeté d'une auto ou comme s'il avait essayé de sauter en marche.

- Il court, il court, le furet, dit Geo. Kauffman barbote l'objet. Un autre arrive, qui le fauche à son tour...

- ... et vous allez le lui refaucher, conclut le Vieux.

- Mais comment donc ! renauda Geo. Pas plus difficile que ça ! Il avait des papiers, le Kauffman?

- Oui. Au nom de Callezan.

- La presse est au courant?

- Pas encore.

Le Gorille, mains dans les poches, arpentait le bureau et le plancher grinçait atrocement.

${ }^{1}$ Désigne les Allemands de l'Ouest. 
- Ça ne mène à rien de penser avec les pieds, remarqua le Vieux, suavement.

Le Gorille s'arrêta et regarda le Vieux.

- Il y a une Marguerite, dans les fichiers?

- Non.

- Merci.

Il reprit sa promenade d'ours, jusqu'à ce que le Vieux exaspéré, se boucha les oreilles. Alors il s'assit de nouveau sur le coin de la table et pointa un index gros comme un canon de fusil vers la poitrine du Vieux.

- Vous allez passer un coup de tube à votre distingué collègue de la Sûreté. Les journaux diront que Callezan a été blessé et qu'il a été hospitalisé dans la salle des urgences... Il n'a pas encore repris connaissance, mais on espère le sauver... Bien entendu, il s'agira d'un fait divers tout ce qu'il y a de banal.

Le Vieux sourit et eut soudain l'air d'un lézard préhistorique.

- C'est déjà fait, dit-il. Seulement, au lieu de l'hôpital, j'ai choisi une clinique, celle du Dr Picard...

Les deux hommes s'observèrent en silence.

- Vous pensez que l'objet est encore en France ? dit Geo.

- Pour les mêmes raisons que vous, dit le Vieux.

Joie de se comprendre à demi-mot.

- Le Dr Picard est d'accord, conclut le Vieux. Avec un peu de chance, vous pourrez prendre le Mistral demain soir.

Le placard était un peu juste. Il puait l'encaustique et le Gorille avait des crampes. Assis sur le derrière, les bras croisés autour des genoux, il somnolait. Si Chaboute lui avait demandé «A quoi penses-tu ? », il aurait pu répondre : A rien. La porte du placard était légèrement poussée. Par l'entrebâillement, le Gorille apercevait le lit où Kauffman était couché. Le piège était appâté. Avec de la viande morte, comme il se doit. Mais le gibier ne venait pas vite. Et s'il ne venait pas ?... Le Gorille bâilla, changea de position ; les fourmis passèrent de sa jambe droite dans sa jambe gauche. Comment serait-elle roulée, cette mystérieuse Margot ? J'ai tout du cocu, dans ce placard, songea le Gorille. Heureusement que je vais la coincer... La coincer... Touchons du bois... bois de campêche... pêche à la ligne... ligne de fond $\ldots$ fonds de commerce... commerce de bois... bois de campêche... Et puis merde... Qu'est-ce que c'est ?...

Rien. Simplement Kauffman qui floflopait, bourré de gaz exubérants. Le Gorille chercha un appui pour sa tête, se cala contre les balais suspendus. Charmantes vacances! Vue imprenable, odeurs sylvestres. Un petit coin bien peinard.

Et soudain, il sursauta. Au pied du lit, une ombre se déplaçait lentement, sans bruit. S'était-il endormi ? Ou bien l'autre avait-il traversé la muraille ? La veilleuse jetait un reflet bleu sur la gabardine, sur le poing ganté qui serrait un revolver muni d'un silencieux. La silhouette était mince. Marguerite ? Marguerite habillée en homme ? L'ombre s'approchait de Kauffman. Doucement, les doigts coururent sur le visage du mort. La main qui tenait le revolver s'abaissa, remit l'arme dans la poche de la gabardine. L'ombre sortit du champ. Le Gorille attendit. Ce n'était pas le moment de se faire repérer. Il poussa doucement la porte du placard, jeta un coup 
d'œil. La chambre était vide. Le rodéo commençait. Avec une souplesse prodigieuse, Geo sortit dans le couloir qui filait, désert, jusqu'à l'escalier. Les consignes avaient été soigneusement observées. Pas un gêneur. Il n'y avait plus qu'à cueillir la Marguerite. Geo fonça sur la pointe des pieds. Mais l'ombre fonçait encore plus vite, comme si elle avait le feu aux fesses. Elle était déjà dans la cour ; elle se faufilait devant la loge. Le Gorille piqua un sprint façon jeux Olympiques qui l'amena sur le trottoir, juste à temps pour voir démarrer une espèce de petit hors-bord rageur. Il sauta en voltige dans sa 15 et mit la gomme. Il comprit tout de suite que le rallye n'était pas dans la poche. L'autre tordue roulait à toute berzingue. Elle était seule et là, le Gorille ne pigeait plus. Elle aurait $d u$ être accompagnée, ou alors c'est que les déductions foirottaient sur les bords. Ou bien c'est que la Margot était un homme.

Geo rasa le pare-chocs d'un nuiteux qui regagnait sa station. A force de jouer au con, il allait sûrement décrocher la timbale. Le pont de Neuilly fut avalé dans le style Fangio et le rond-point de la Défense apparut. Le petit bolide ralentit, piqua droit vers le Palais des Expositions.

- Vu, se dit Geo. C'est ici qu'on passe le relais.

Il freina, éteignit ses feux, puis stoppa car la voiture venait de s'arrêter, là-bas, devant une porte surmontée d'une pancarte Entrée des Exposants. Il commençait à ne plus piger du tout, le Geo. Est-ce que, par hasard, il se serait fait avoir ? Est-ce que la Margot s'était taillée en douce, à la sortie de la clinique, pendant qu'un complice attirait les éventuels poursuivants ? Mais non. Pas possible... Kauffman avait mis le nez dans leur pot aux roses. Ça, c'était sûr. Ils l'avaient descendu pour l'empêcher de parler. Bon. D'où le raid sur la clinique. Il fallait que Kauffman soit mort pour que l'expédition de l'accu soit tentée sans risque. La logique, ça existe. Et puisque la tueuse, ou le tueur (pas moyen de préciser encore) avait constaté que Kauffman ne dirait jamais ce qu'il savait, la voie était libre. On allait s'occuper sérieusement de l'objet...

Le Gorille, à demi rassuré, sortit de la 15 comme si le sol avait été semé de tessons de bouteilles. Deux heures. Roupillon général. Silence. L'ombre avait ouvert la porte et, maintenant, sortait du coffre une chose allongée qui ne paraissait pas précisément gonflée d'air. Mais il n'avait pas l'air emprunté, car c'était un homme, pas d'erreur possible. Et il en connaissait un bout sur la manutention. Le colis sur l'épaule, un peu cassé par le poids, malgré tout, il disparut dans le bâtiment. Le Gorille, en quelques bonds, fut devant la porte. Elle n'était même pas fermée. Le temps de la pousser, de se risquer sur les pointes comme une danseuse de l'Opéra... Le sol était en ciment. Un coup de pot. Le Gorille, chaussé de crêpe, était plus silencieux qu'un fantôme, mais se voyait, malheureusement, de plus loin. Le gazier, par chance, était trop absorbé par son boulot pour se retourner. Il suivait bien sagement le couloir, précédé du pinceau de sa lampe électrique. Le couloir bifurqua et l'homme éclaira une pancarte, tourna à gauche, le Gorille dans son sillage. Une autre porte. Geo devenait méfiant. Qu'est-ce qu' on venait foutre dans le Palais des Expositions ? Quel rapport avec les accus de la maison Weber ? ... Geo franchit la dernière porte sur les talons du rombier. Il faillit lâcher un mot percutant. Il s'était attendu à tout sauf à ça. Devant lui, le hall du Palais semblait s'élargir à l'infini. La voûte était noire comme celle d'une forêt. Aux pieds du Gorille, c'était un jardin, un 
vrai, avec des fleurs qui brillaient doucement, éclairées, de loin en loin, par des ampoules dont la lumière, trop faible, faisait ressembler les allées à des ruelles d'ombre vaguement bariolées. "Les Floralies», pensa Geo. C'était tellement incongru qu'il s'arrêta une seconde, saisi d'une formidable envie de se marrer. "Ça manque un peu d'oiseaux !», songea t-il encore, avant de reprendre sa poursuite. Ce n'était même plus une poursuite, mais une petite balade du genre footing matinal, à la fraîche. Pas un gardien en vue. Ils devaient en écraser quelque part, abrutis par les odeurs puissantes qui flottaient sous la voûte, dans un silence à couper au couteau. Un truc à attraper un rhume des foins. La barbouze trottait toujours, son butin sur l'épaule, parmi les tulipes et les azalées. Il contourna un massif de dahlias, longea un carré de quelque chose qui sentait le gazon mouillé et suivit une allée spacieuse, bordée de petits arbres, car il y avait aussi des arbres. Le Gorille était résolu à ne plus s'étonner. Peut-être allait-il croiser une vache ou un troupeau de moutons. Des panneaux, qui paraissaient suspendus dans le vide, marquaient les frontières. Geo apprenait ainsi qu'il venait de quitter les Pays-Bas pour la Suisse et qu'il allait pénétrer aux U.S.A. Devant, le globe-trotter se maniait drôlement le train. Il traversa les U.S.A. à la vitesse d'une soucoupe volante, entra dans le stand de l'U.R.S.S. Intéressé, Geo se rapprocha, en s'abritant derrière un rideau de palmiers. L'homme avait posé son colis sur le sol et attrapé une pelle à manche court qui, comme par hasard, était plantée au coin d'un parterre. Et ce parterre, vaste comme une prairie, était un champ de marguerites. Mais pas des marguerites comme vous et moi. Des marguerites revues et corrigées, à cœur blanc et pétales noirs, des marguerites de choc, boulottant de la vitamine irradiée, de l'hormone sélectionnée, du fumier atomique. Le gonze en mettait un coup. Les fleurs étaient plantées dans de longues caisses basses, du type jardinière, qui étaient rangées côte à côte. Avec sa pelle, il enlevait bien proprement la terre, installait avec des précautions de nourrice terreau et fleurs sur le ciment de l'allée. La première caisse, bientôt vidée, était assez profonde pour recevoir l'accumulateur. Marguerite ! Le Gorille commençait à saisir le coup. Pas question d'une fillette ! C'était beaucoup plus fortiche. On collait l'objet sous les fleurs et, après les Floralies, ces messieurs rapatriaient leur précieuse camelote, à la barbe des barbouzes. Pas touche. Fleurs scientifiques. Voyageant en franchise, avec la garantie du gouvernement. C'était pratique et touchant. Le Rousky s'était relevé pour s'éponger le front. Geo s'avança vers lui, mais, peut-être alerté par son sixième sens, l'homme se retourna brusquement. Déjà, il avait sorti son revolver à silencieux. Geo leva les bras, docilement, ses mains se perdant parmi les feuilles raides d'un palmier. Le Popoff fit deux pas. Le troisième resta inachevé. Geo avait empoigné le sommet du palmier et, se baissant avec la violence et la vitesse d'un ressort, il avait arraché l'arbre, d'un coup de reins. Le palmier, crachant des mottes à tous les azimuts, décrivit un arc de cercle foudroyant et ses racines, renflées comme l'extrémité d'une massue, rebondirent sur la tète du rombier qui s'effondra pour le compte. Alors le Gorille se transforma en jardinier modèle. D'abord, le palmier. En quelques secondes, il réintégra sa plate-bande. La terre, tassée à la pelle, reprit un aspect débonnaire. Ce fut ensuite le tour du cadavre, dont les poches ne contenaient rien d'intéressant. Geo le coucha dans la caisse, et entreprit de le recouvrir de marguerites. Accroupi comme un moufflet en train de faire des pâtés, il remodelait le 
parterre à la main, et peu à peu lui rendait son aspect maison. A la fin, il n'en pouvait plus, mais les marguerites avaient vraiment bon air. Geo remonta son pantalon, alluma une Gauloise qui sentait la mousse et le sous-bois et, l'accu sous le bras, gagna la sortie paisiblement, comme un qui vient de finir sa journée.

Une dizaine de jours plus tard, le Gorille était de retour avec le Vieux. Des équipes d'ouvriers démontaient les stands, enlevaient les fleurs. Quelques « officiels », des journalistes, des photographes, erraient au hasard des allées jonchées de débris. Autour des marguerites, des curieux s'attardaient. Un photographe prenait quelques clichés. Le Vieux, à petits pas, se promenait.

- Curieux, dit-il au Gorille... Tous ces palmiers pètent de santé, sauf celui-là... Il est tout jaune, tout fané...

- Taisez-vous, grommela Geo. Ils ont peut-être des hommes à eux.

- Et ces marguerites, reprit le Vieux. Comme c'est drôle... Elles sont toutes abîmées, défraîchies... sauf ce carré.

Il se baissa, cueillit une marguerite dont il regarda les pétales machinalement.

- Vous avez bonne mine, chuchota Geo.

Des ouvriers les firent reculer et soulevèrent la caisse qu'ils portèrent jusqu'à un camion bâché.

Passionnément... Pas du tout..., murmura le Vieux.

- Amen, conclut le Gorille.

\section{Bibliographie}

[AMR 98] AMREIN M., Lire des textes littéraires au cycle III, Stage de formation départementale, Clermont-Ferrand, 22-26 septembre 1997, CRDP d'Auvergne, 1998.

[AUF 96] AufFRET GWENDAL, «LECAO », Etats généraux de l'écriture multimédia, NOV'ART spécial, février 1996.

[BAR 70] BARTHes R., S/Z, Seuil, Paris, 1970.

[BAR 73] BARTHES R., « Analyse textuelle d'un conte d'Edgar Poe », Sémiotique narrative et textuelle, Chabrol C., Collection L, Librairie Larousse, Paris, p. 7-57, 1973.

[BER 99] BERNARD M., Introduction aux études littéraires assistées par ordinateur, PUF, collection écriture électroniques, Paris, 1999.

[BES 93] Besiat J.-P., «Texte, champs lexicaux et HyperTexte », CALIBAN, Presses universitaires du Mirail, Toulouse, $n^{\circ}$ 30, p. 117-136, 1993.

[BOI 80] Boileau P., Narcejac T., Usurpation d'identité, Hachette, Collection J'ai Lu, Paris, 1980.

[BRU 97] BRUILLARD E., Les machines à enseigner, Hermès, Paris, 1997. 
[CLE 98] CLEMENT J., « Du livre au texte», in Le livre électronique, Sciences et techniques éducatives, volume 5, n 4, Hermès, décembre 1998.

[COI 96] Coirier P., Gaonac'h D., Passerault J-M., Psycholinguistique textuelle, Approche cognitive de la compréhension et de la production des textes, Armand Colin, Paris, 1996.

[CRI 97] CRINON J., PACHET S., «Des ressources hypertextes pour écrire au cycle 3 », in Apprendre avec le multimédia, Céméa, Retz, Paris, 1997.

[DUB 95] Dubois C., «Apport de l'informatique à l'enseignement littéraire en vue des nouvelles épreuves du baccalauréat », EPI, n 80, décembre 1995, p. 73-79, 1995.

[DUB 98] DuboIS C., «Initier à la lecture méthodique en seconde à l'aide de l'informatique », EPI, n 92 , Décembre 1998.

[ECO 85] Eco U., Lector in fabula, Grasset, Le livre de Poche, , Paris, 1985.

[FOU OO] Fourtanier, M.-J., LAnglade G., Enseigner la littérature, Actes du colloque Enjeux didactiques des théories du texte dans l'enseignement du français, 19-21 fécrier 1998, IUFM de Toulouse, CRDP Midi-Pyrénées/Delagrave, Toulouse, 2000.

[GOD 98] Godinet H., Lire-écrire des hypertextes, Thèse de $3^{\text {ème }}$ cycle, Grenoble, 1998.

[GOO 79] Goody J., La raison graphique, Les Éditions de Minuit, Paris, 1979.

[GRE 93] Greimas A. J., Courtes J., Sémiotique, dictionnaire raisonné de la théorie du langage, Hachette supérieur, 1993.

[ISE 97] ISER W., L'acte de lecture, théorie de l'effet esthétique, Mardaga, Collection Philosophie et langage, 1997.

[JAU 78] Jauss H. R., Pour une esthétique de la réception, Gallimard, Paris, 1978.

[JAK 63] JAKOBSON R., Essais de linguistique générale, T. 1, chapitres II, IX, XI, Les éditions de minuit, Paris, 1963.

[KER 77] KeRbrat-Orecchioni C., La connotation, Presses universitaires de Lyon, 1977.

[LEB 97] LeBrave J-L., «Hypertexte et édition génétique: l'exemple d'Hérodias de Flaubert », Banques de données et hypertextes pour l'étude du roman, FERRAND N., PUF, Collection écritures électroniques, 1997.

[LEV] LEVY P., «La virtualisation du texte», http://hypermedia.univparis8.fr/pierre/virtuel/virt3.htm

[MAN 96] MANGENOT F., Les aides logicielles à l'écriture, CNDP, Collection de l'Ingénierie éducative, Paris, 1996.

[MAZ 95] MaZhoud O., Pascual E., ViRbel J., "Représentations et gestion d'annotations", Hypertextes et hypermédias, Actes de la 3ème Conférence de l'Université Paris 8, J.-P. Balpe, A. Lelu, I. Saleh, Hermès, 1995.

[NEV 85], NEVEU E., L'idéologie dans le roman d'espionnage, Presses de la Fondation nationale des Sciences Politiques, Paris, 1985.

[ORI 86] ORIOL-BOyER C., « Lire/écrire avec des enfants (I) », TEM n 5, Printemps 1986. 
[ORI 88a] ORIOL-BOYER C., « De l'usage ordinaire à l'usage artistique de l'écriture : activité « méta » et didactique », Études de Linguistique Appliquée, nº 71, p. 70-85, 1988.

[ORI 88b] ORIOL-BOyer C., L'écriture du texte (Théorie, Pratique, Didactique), Thèse d'État, Grenoble, 1988.

[ORI 98] Oriol-Boyer C., Driol M., Oriol J-C., SAMY T., « hypertexte et didactique de la production textuelle en français », EPI, n 89 , Mars 1998.

[SOU 01] SoubriE T., Apprendre à lire grâce à l'hypertexte, Thèse de $3^{\text {ème }}$ cycle, Montpellier, 2001.

[TAU 96] TAUVERON C., REUTER Y., Lecture et écriture littéraires à l'école, Repères, $\mathrm{N}^{\circ} 13$, Nouvelle série, INRP, Paris, 1996.

[VUI 99] Vuillemin A., «La lecture informatique et l'écrilecture », Littérature, informatique, lecture, Vuillemin A., Lenoble M., Presse Universitaire de Limoges, Limoges, p. 101-110, 1999. 